\title{
Factors Associated with Klebsiella Bacteremia and Its Outcome in Under-Five Children Admitted with Diarrhea
}

\author{
Shamima Akhter, Tahmeed Ahmed, Shafiqul Alam Sarker, Monira Sarmin, \\ Abu S. M. S. B. Shahid, K. M. Shahunja, Shoeb Bin Islam, Lubaba Shahrin, Tahmina Alam, \\ Nur Haque Alam, and Mohammod Jobayer Chisti
}

Nutrition and Clinical Services Division (NCSD), International Centre for Diarrhoeal Disease Research, Bangladesh (icddr,b), Dhaka 1212, Bangladesh

Correspondence should be addressed to Mohammod Jobayer Chisti; chisti@icddrb.org

Received 12 March 2016; Accepted 1 August 2016

Academic Editor: F. J. Kaskel

Copyright ( 2016 Shamima Akhter et al. This is an open access article distributed under the Creative Commons Attribution License, which permits unrestricted use, distribution, and reproduction in any medium, provided the original work is properly cited.

\begin{abstract}
Although Klebsiella bacteremia in children is perceived to be associated with fatal consequences, data are scarce on those children presenting with diarrhea. We evaluated the factors associated with Klebsiella bacteremia in such children. In this retrospective chart analysis, data of all diarrheal children was retrieved from electronic medical record system (named as SHEBA) of Dhaka Hospital of International Centre for Diarrhoeal Disease Research, Bangladesh (icddr,b), from January 1, 2010, to December 31, 2012, who had their blood culture done. This was a study having a case-control design where comparison of clinical and laboratory characteristics was done among children with Klebsiella bacteremia (cases $=30$ ) and those without any bacteraemia (controls $=90)$. Controls were selected randomly. The cases more often had fatal outcome $(p<0.001)$. In logistic regression analysis, after adjusting for potential confounders such as young age, severe dehydration, severe wasting, abnormal mentation, hypotension, and fast breathing, the cases were independently associated with hospital-acquired infection and positive stool growth (for all, $p<0.05$ ). The study highlights the importance of obtaining blood cultures in hospitalized children under five years old with diarrheal illness in the presence of either hospital-acquired infection or positive stool culture to have better outcome.
\end{abstract}

\section{Introduction}

Diarrhea still remains as one of the leading killer diseases of children under five in developing countries and accounts for $9 \%$ of 6.3 million global deaths in 2013 [1]. Death is even higher in diarrheal children having bacteremia compared to those without [2]. An earlier report [3] from icddr,b has shown factors that were associated with an increased risk of death in bacteremic patients who were infected with a Gram-negative pathogen; however, among the Gramnegative bacteremia, Klebsiella is one of the most virulent pathogens and is often associated with high morbidity and mortality in children [3]. It has also been found to be the most common cause of pneumonia in severely malnourished children [4]. Klebsiella bacteremia is perceived to be more common in diarrheal children compared to those without diarrhea and often have fatal outcomes. In resource-poor settings, where laboratory facilities are limited and blood culture is seldom done, clinical features may help in predicting Klebsiella bacteremia in such children. However, to our knowledge, there is no published data on the role of Klebsiella bacteremia and its clinical features in diarrheal children. The objective of our study was to evaluate the factors associated with Klebsiella bacteremia in under-five diarrheal children and their outcome.

\section{Materials and Methods}

2.1. Ethical Statement. This study was solely a medical record analysis. This study did not involve any interviews with patients or care givers. Data were anonymous before analysis.

2.2. Study Site. The study population was treated in the Dhaka Hospital of International Centre for Diarrhoeal Disease Research, Bangladesh (icddr,b) and the description of the study site has been provided elsewhere [5]. 
2.3. Study Design. All the diarrheal children under five who had their blood culture done between January 1, 2010, and December 31, 2012, were enrolled in the study. A casecontrol design was deployed in the study where the children having Klebsiella bacteremia constituted the cases and those without any bacteremia constituted the controls. Thus, we did not include the children with positive blood cultures for organisms other than Klebsiella species as potential controls. We had taken threefold controls using randomization process in the Statistical Package for Social Science (SPSS) to increase the power of our analysis.

2.4. Patient Management. Management of the patients has been done following standardized protocol followed in the Dhaka Hospital of icddr,b and the management of hospitalized patient has also been described elsewhere [5].

2.5. Data Collection and Measurements. Data of all diarrheal children was retrieved from electronic medical record system (named as SHEBA) of Dhaka Hospital. After admission, every patient received a unique identification number. All the data were recorded under this number. Case report forms (CRF) were developed for this study, pretested, and finalised for data acquisition. Characteristics analyzed included demographics (age, gender), nutritional status including severe wasting $(z$ score for weight for length/height $<-3$ of WHO growth standard) and severe underweight ( $z$ score for weight for age $<-3$ of WHO growth standard), abnormal mentation (drowsiness, convulsion, or restlessness), fast breathing $(<2$ months: $\geq 60 / \mathrm{min} ; 2-<12$ months: $\geq 50 /$ min; $12-59$ months: $\geq 40 / \mathrm{min}$ ), $\mathrm{SpO}_{2}$ (transcutaneously measured blood oxygen concentration), dehydration status, hypotension (defined as systolic blood pressure $\leq 70 \mathrm{~mm}$ of $\mathrm{Hg}$ or diastolic blood pressure $\leq 40 \mathrm{~mm}$ of $\mathrm{Hg}$ or mean arterial pressure $\leq 50 \mathrm{~mm}$ of $\mathrm{Hg}$ ) [6], hospital-acquired infection (new episode of infection at least after 48 hours of hospitalization), laboratory investigation (creatinine in micro-mol/L, stool culture for Vibrio cholerae, Shigella species, Salmonella typhi, and other vibrios), and outcome (deaths).

2.6. Statistical Analysis. All data were entered into a personal computer and edited before analysis using SPSS for Windows (version 17.0; SPSS Inc., Chicago, IL, USA) and Epi Info (version 6.0; USD, Stone Mountain, GA, USA). Differences in proportions were compared by the Chi-square test. In normally distributed data, differences in means were compared by Student's $t$-test, and the Mann-Whitney test was used for comparing data that were not normally distributed. A probability of less than 0.05 was considered statistically significant. Strength of association was determined by calculating odds ratio (OR) and their 95\% confidence intervals (CIs). To identify clinical predictors associated with Klebsiella bacteremia in diarrheal children, variables were initially analyzed in a univariate model, and then, after adjusting for potential confounders, a multiple logistic regression model was used to identify the independent predictors of Klebsiella bacteremia.

\section{Results}

During the 3-year study period, a total of 3313 children fulfilled the study criteria and we only identified 30 (0.9\%) cases of Klebsiella bacteremia. Among the remaining 3283 children, 676 had bacterial isolates other than Klebsiella species. Thus, we had randomly selected 90 controls among a total of 2607 children who had no growths in their blood culture and were available for the selection of the controls. Diarrheal children with Klebsiella bacteremia more often presented at their infancy, received antibiotics prior to admission, and had severe dehydration, fever, abnormal mentation, severe wasting, and positive stool growth on admission and they frequently developed hospital-acquired infection and more often had fatal outcome during hospitalization compared to those without bacteremia (Table 1). Other variables in Table 1 were comparable among the groups. In logistic regression analysis, after adjusting for potential confounders such as young age, severe dehydration, severe wasting, abnormal mentation, hypotension, and fast breathing, the cases were independently associated with hospital-acquired infection and positive stool growth (Table 2). Importantly, Klebsiella species causing bacteremia was found to have higher resistant (68\%) to ceftriaxone. Bacterial isolates from stool have been shown in Table 3.

\section{Discussion}

Gram-negative bacteremia is a major cause of morbidity and mortality if undiagnosed on time $[7,8]$. The observation of higher case fatality rate in children with Klebsiella bacteremia compared to those without any bacteremia was expected. We are not aware of any report on mortality due to Klebsiella bacteremia in diarrheal children. However, a number of previous studies in nondiarrheal children revealed that children with Gram-negative bacteremia more often had fatal outcomes compared to those without bacteremia $[7,8]$.

In this study, we observed that the prevalence of Klebsiella bacteremia is very low in children with diarrhea. However, in a number of hospital based studies, a higher prevalence was demonstrated that ranged from $13 \%$ [8] to $26 \%$ [9]. In a study from Ghana, 26\% Klebsiella bacteremia was detected in children below 1 month of age [9]. Also in the same age group reports from Nigeria, Ghana, and the United States, Klebsiella accounts for 3 to $7 \%$ of all nosocomial bacterial infections [10]. The potential reasons for variations in prevalence of Klebsiella bacteremia might be due to heterogeneity in geographical location, patient population, study design, or differences in prior antibiotic therapy. Moreover, all of our study children had diarrhea which was not present in those above-mentioned studies [10] and that might be one of the reasons of variation in prevalence of Klebsiella bacteremia in our study children. To our knowledge this is the first study that reported the data on Klebsiella bacteremia in underfive diarrheal children from Bangladesh. One of the plausible reasons for the development of bacteremia in diarrheal children might be due to bacterial translocation through transcellular and paracellular pathways. It mostly occurs in immune compromised children and impairment of normal 
TABLE 1: Characteristics of under-five diarrheal children with (cases) and without (controls) Klebsiella bacteremia at hospitalization and their outcome during hospitalization.

\begin{tabular}{|c|c|c|c|c|}
\hline Characteristics & $\begin{array}{c}\text { Culture positive } \\
\begin{array}{c}n=30 \\
(\%)\end{array}\end{array}$ & $\begin{array}{l}\text { Culture negative } \\
\begin{array}{c}n=90 \\
(\%)\end{array}\end{array}$ & OR $(95 \% \mathrm{CI})$ & $p$ \\
\hline Young age (<12 months) & $24(80)$ & $53(59)$ & $2.79(1.00-8.5)$ & 0.037 \\
\hline Male & $19(63)$ & $53(60)$ & $1.14(0.45-2.93)$ & 0.933 \\
\hline $\begin{array}{l}\text { Duration of diarrhea in days } \\
\text { (median IQR) }\end{array}$ & $4(2.00-7.00)$ & $3(2.00-5.00)$ & - & 0.193 \\
\hline Antibiotics prior to admission & $28(97)$ & $67(78)$ & $0.13(0.01-0.97)$ & 0.05 \\
\hline Severe dehydration & $6(20)$ & $4(5)$ & $5.25(1.18-24.56)$ & 0.017 \\
\hline Fever $\left(\geq 38^{\circ} \mathrm{C}\right)$ & $8(27)$ & $47(53)$ & $0.32(0.14-0.87)$ & 0.023 \\
\hline Abnormal mentation & $25(83)$ & $41(57)$ & $5.48(1.92-15.67)$ & $<0.001$ \\
\hline Systolic BP $($ mean \pm SD $)$ & $86 \pm 13$ & $93 \pm 19$ & - & 0.218 \\
\hline Diastolic BP $($ mean \pm SD $)$ & $53 \pm 11$ & $55 \pm 14$ & - & 0.799 \\
\hline Hypotension & $3 / 17(18)$ & $5 / 29(17)$ & $1.03(0.16-6.15)$ & 1.00 \\
\hline Fast breathing & $9(30)$ & $46(52)$ & $0.4(0.15-1.05)$ & 0.064 \\
\hline $\mathrm{SpO}_{2}($ mean $\pm \mathrm{SD})$ & $96.70 \pm 3.08$ & $96.56 \pm 3.41$ & - & 0.847 \\
\hline Creatinine $($ mean $\pm \mathrm{SD})$ & $49.80 \pm 39.59$ & $42.80 \pm 45.91$ & - & 0.250 \\
\hline Severe wasting & $13(59)$ & $19(26)$ & $4.18(1.39-12.81)$ & 0.007 \\
\hline Severe underweight & $21(72)$ & $46(55)$ & $2.16(0.8-6.04)$ & 0.151 \\
\hline Vibrio cholerae & $1(3)$ & $3(3)$ & $0.33(0.01-3.92)$ & 0.614 \\
\hline Shigella & $5(17)$ & $5(6)$ & $0.34(0.08-1.4)$ & 0.132 \\
\hline Positive stool culture & $13(43)$ & $10(11)$ & $6.12(2.08-18.28)$ & $<0.001$ \\
\hline HAI & $11(37)$ & $2(2)$ & $25.47(5.21-124.43)$ & $<0.001$ \\
\hline Death & $12(40)$ & $3(3)$ & $19.33(4.41-97.31)$ & $<0.001$ \\
\hline
\end{tabular}

Figures represent $n$ (total number), unless specified. OR: odds ratio. CI: confidence interval. IQR: interquartile range. SD: standard deviation. SpO ${ }_{2}$ : transcutaneously measured blood oxygen concentration. HAI: hospital acquired infection.

TABLE 2: Results of logistic regression analysis to explore the independent association of Klebsiella bacteremia in diarrheal children under five years of age.

\begin{tabular}{lccc}
\hline Characteristics & OR & $95 \%$ CI & $p$ \\
\hline Young age (<12 months) & 4.72 & $0.06-349.48$ & 0.479 \\
Severe wasting & 10.26 & $0.57-185.56$ & 0.115 \\
Positive stool culture & 26.51 & $1.05-671.23$ & 0.047 \\
Abnormal mentation & 0.36 & $0.005-24.54$ & 0.633 \\
Severe dehydration & 24.15 & $0.44-1310.45$ & 0.118 \\
HAI & 307.1 & $2.16-43658.91$ & 0.024 \\
Hypotension & 20.50 & $0.39-1073.92$ & 0.135 \\
Fast breathing & 0.02 & $0.00-1.47$ & 0.075 \\
\hline
\end{tabular}

ecological balance of gut, mucosal barrier permeability, and stress [11].

In this study, we found an independent association of hospital-acquired infection and positive stool growth with Klebsiella bacteremia. On the other hand, young age, severe dehydration, severe wasting, and abnormal mentation were associated with Klebsiella bacteremia in a univariate analysis, although, in logistic regression analysis, their association with Klebsiella bacteremia became insignificant. While hypotension and fast breathing are the components of sepsis in adults following qSOFA criteria [12] and both in adults and
TABLE 3: Bacterial isolates from stool culture.

\begin{tabular}{lcc}
\hline Organism isolated & $\begin{array}{c}\text { Cases } \\
(n=13) \\
(\%)\end{array}$ & $\begin{array}{c}\text { Controls } \\
(n=10) \\
(\%)\end{array}$ \\
\hline Vibrio cholerae & $1(3)$ & $3(3)$ \\
Shigella species & $5(17)$ & $5(6)$ \\
Salmonella typhi & 0 & $1(1)$ \\
Other vibrios & $2(7)$ & $1(1)$ \\
Other organisms & $5(17)$ & 0 \\
\hline
\end{tabular}

children following Surviving Sepsis Guidelines [13], these two variables did not have significant association with Klebsiella bacteremia in our study children either by a univariate analysis or a logistic regression.

The US National Healthcare Safety Network indicates that infection due to Gram-negative bacteria is responsible for more than $30 \%$ of hospital-acquired infections. Klebsiella was found to be very common organism responsible for such type of infection in children [14].

To our knowledge, the observation of an independent association of Klebsiella bacteremia with bacterial isolates from diarrheal stool in our study has been described for the first time in medical literature. We do not have any ready explanation for this observation but altered gut function, 
mucosal damage, enteropathy, or absence of antibacterial peptide or loss of bacterial flora in diarrhea may have influences on translocation of Klebsiella from the gut [11, 15]. We have also evaluated sensitivity for extended spectrum beta-lactam (ESBL) antibiotics among Klebsiella species and in our reference laboratory, the surrogate marker of ESBL is ceftriaxone; Klebsiella species was found to have higher resistance $(68 \%)$ to ceftriaxone. The finding is consistent with previous observation [16].

Gram-negative bacteremia is relatively common in young age and occurs much less frequently after the first year of life. Infants had the highest incidence of Gram-negative bacteremia among all children observed in a previous study [17], which is consistent with our study and Klebsiella was one of the commonest organisms isolated [17]. The increased incidence of Klebsiella bacteremia in young age might be due to less cellular and humoral immunity.

Wasting is an important indicator of acute malnutrition which impairs the immune response and predispose to invasive infection. Comorbidity of diarrhea with malnutrition makes the child more vulnerable to invasive infection $[11,18]$. A number of previous studies [19-21] have been shown to have significant association of severe acute malnutrition with Klebsiella bacteremia.

Abnormal mentation as observed in this study was an associated factor with Klebsiella bacteremia which may also be common in any bacteremia and might be due to compromised perfusion or systemic response to Klebsiella bacteremia which is consistent with other studies with any bacteremia [22].

The main limitation is the retrospective nature of the study which involves a small number of samples that limited the power of the study and subsequently limited the generalisability of the study results. Another limitation is the lack of specification of Klebsiella species into $K$. pneumoniae and $K$. oxytoca.

In conclusion, the prevalence of Klebsiella bacteremia in diarrheal children was less than $1 \%$. Diarrheal children under five with Klebsiella bacteremia had greater case fatality rate compared to those without bacteremia. The study highlights the importance of obtaining blood cultures in hospitalized children under five years old with diarrheal illness in the presence of either infancy, abnormal mentation, severe wasting, severe dehydration, hospital-acquired infection, or positive stool culture, although hospital-acquired infection and positive stool culture were identified as the independent risk factors for Klebsiella bacteremia in our study population. Awareness and identification of these simple clinical characteristics may help in early case detection and management of Klebsiella bacteremia and therefore help in reducing deaths in such children from developing countries. However, further research in diarrheal children with Klebsiella bacteremia with larger sample is warranted to substantiate our observation.

\section{Disclosure}

The funders had no role in study design, data collection and analysis, decision to publish, or preparation of the paper.

\section{Competing Interests}

The authors declare that they have no competing interests.

\section{Acknowledgments}

This research was funded by core donors which provide unrestricted support to icddr,b for its operations and research. Current donors providing unrestricted support include Government of the People's Republic of Bangladesh; Global Affairs Canada (GAC); Swedish International Development Cooperation Agency (Sida), and the Department for International Development (UK Aid). The authors gratefully acknowledge these donors for their support and commitment to icddr,b's research efforts. They would like to express their sincere thanks to all physicians, clinical fellows, nurses, members of the feeding team and cleaners of the hospital, and SHEBA team for their invaluable support and contribution for patient care and data collection.

\section{References}

[1] UNICEF, "Committing to child survival: a promise renewed," Progress Report, 2014.

[2] L. Liu, S. Oza, D. Hogan et al., "Global, regional, and national causes of child mortality in 2000-13, with projections to inform post- 2015 priorities: an updated systematic analysis," The Lancet, vol. 385, no. 9966, pp. 430-440, 2015.

[3] B. L. Meatherall, D. Gregson, T. Ross, J. D. D. Pitout, and K. B. Laupland, "Incidence, risk factors, and outcomes of klebsiella pneumoniae bacteremia," American Journal of Medicine, vol. 122, no. 9, pp. 866-873, 2009.

[4] M. J. Chisti, M. Tebruegge, S. La Vincente, S. M. Graham, and T. Duke, "Pneumonia in severely malnourished children in developing countries-mortality risk, aetiology and validity of WHO clinical signs: a systematic review," Tropical Medicine \& International Health, vol. 14, no. 10, pp. 1173-1189, 2009.

[5] M. J. Chisti, M. A. Salam, J. H. Smith et al., "Bubble continuous positive airway pressure for children with severe pneumonia and hypoxaemia in Bangladesh: an open, randomised controlled trial," The Lancet, vol. 386, no. 9998, pp. 1057-1065, 2015.

[6] K. Maitland, S. Kiguli, R. O. Opoka et al., "Mortality after fluid bolus in African children with severe infection," The New England Journal of Medicine, vol. 364, no. 26, pp. 2483-2495, 2011.

[7] M. J. Chisti, S. M. Graham, T. Duke et al., "A prospective study of the prevalence of tuberculosis and bacteraemia in Bangladeshi children with severe malnutrition and pneumonia including an evaluation of Xpert MTB/RIF assay," PLoS ONE, vol. 9, no. 4, Article ID e93776, 2014.

[8] A. G. Falade, I. A. Lagunju, R. A. Bakare, A. A. Odekanmi, and R. A. Adegbola, "Invasive pneumococcal disease in children aged $<5$ years admitted to 3 urban hospitals in Ibadan, Nigeria," Clinical Infectious Diseases, vol. 48, no. 2, pp. S190-S196, 2009.

[9] M. V. Nielsen, N. Sarpong, R. Krumkamp et al., "Incidence and characteristics of bacteremia among children in rural Ghana," PLoS ONE, vol. 7, no. 9, Article ID e44063, 2012.

[10] R. Podschun and U. Ullmann, "Klebsiella spp. as nosocomial pathogens: epidemiology, taxonomy, typing methods, and pathogenicity factors," Clinical Microbiology Reviews, vol. 11, no. 4, pp. 589-603, 1998. 
[11] C. McKiernan, L. C. Chua, P. F. Visintainer, and H. Allen, "High flow nasal cannulae therapy in infants with bronchiolitis," Journal of Pediatrics, vol. 156, no. 4, pp. 634-638, 2010.

[12] M. Singer, C. S. Deutschman, C. W. Seymour et al., "The third international consensus definitions for sepsis and septic shock (sepsis-3)," The Journal of the American Medical Association, vol. 315, no. 8, pp. 801-810, 2016.

[13] R. P. Dellinger, M. M. Levy, A. Rhodes et al., "Surviving sepsis campaign: international guidelines for management of severe sepsis and septic shock: 2012," Critical Care Medicine, vol. 41, no. 2, pp. 580-637, 2013.

[14] A. Y. Peleg and D. C. Hooper, "Hospital-acquired infections due to gram-negative bacteria," The New England Journal of Medicine, vol. 362, no. 19, pp. 1804-1813, 2010.

[15] J. MacFie, "Current status of bacterial translocation as a cause of surgical sepsis," British Medical Bulletin, vol. 71, pp. 1-11, 2004.

[16] A. Christopher, S. E. Mshana, B. R. Kidenya, A. Hokororo, and D. Morona, "Bacteremia and resistant gram-negative pathogens among under-fives in Tanzania," Italian Journal of Pediatrics, vol. 39, article 27, 2013.

[17] M. N. Al-Hasan, W. C. Huskins, B. D. Lahr, J. E. Eckel-Passow, and L. M. Baddour, "Epidemiology and outcome of Gramnegative bloodstream infection in children: A Population-Based Study," Epidemiology and Infection, vol. 139, no. 5, pp. 791-796, 2011.

[18] M. J. Chisti, T. Duke, C. F. Robertson et al., "Co-morbidity: exploring the clinical overlap between pneumonia and diarrhoea in a hospital in Dhaka, Bangladesh," Annals of Tropical Paediatrics, vol. 31, no. 4, pp. 311-319, 2011.

[19] D. J. A. Berkley, Invasive Bacterial Infections in Children at a sub-Saharan District Hospital, 2002.

[20] M. A. Adedoyin and D. Fagbule, "Bacterial aetiology of childhood pneuonia," Nigerian Journal of Paediatrics, vol. 14, no. 2, pp. 37-40, 1987.

[21] W. I. Aderele, K. Osinusi, D. Gbadero, F. A. Adeyei-Doro, N. A. Rotowa, and O. A. Okubanjo, "Acute klebsiella, pseudoonas and proteus pneumonia in childhood," Nigerian Journal of Paediatrics, vol. 19, no. 4, pp. 80-88, 1992.

[22] M. Sarmin, T. Ahmed, P. K. Bardhan, and M. J. Chisti, "Specialist hospital study shows that septic shock and drowsiness predict mortality in children under five with diarrhoea," Acta Paediatrica, vol. 103, no. 7, pp. e306-e311, 2014. 


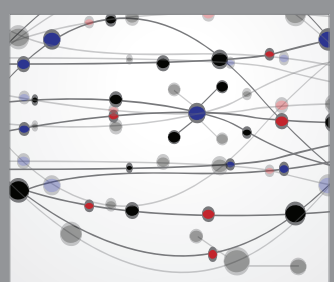

The Scientific World Journal
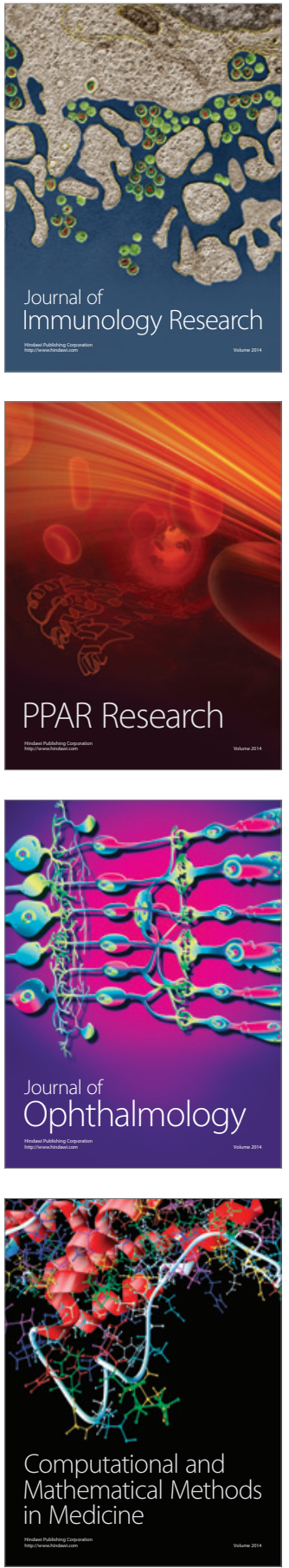

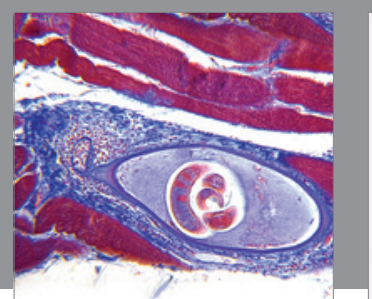

Gastroenterology Research and Practice

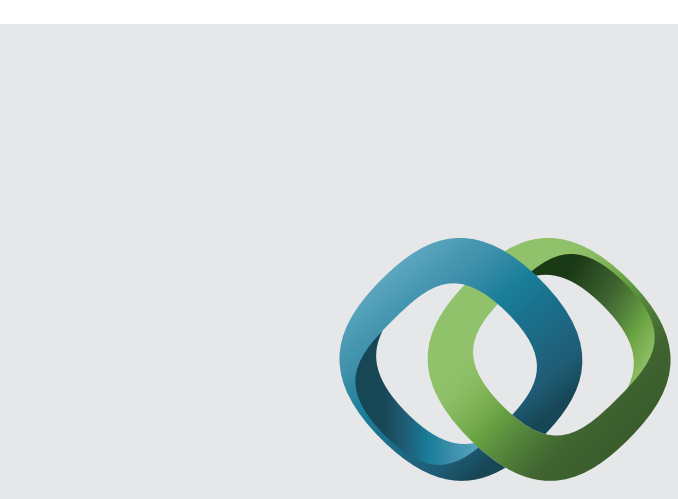

\section{Hindawi}

Submit your manuscripts at

http://www.hindawi.com
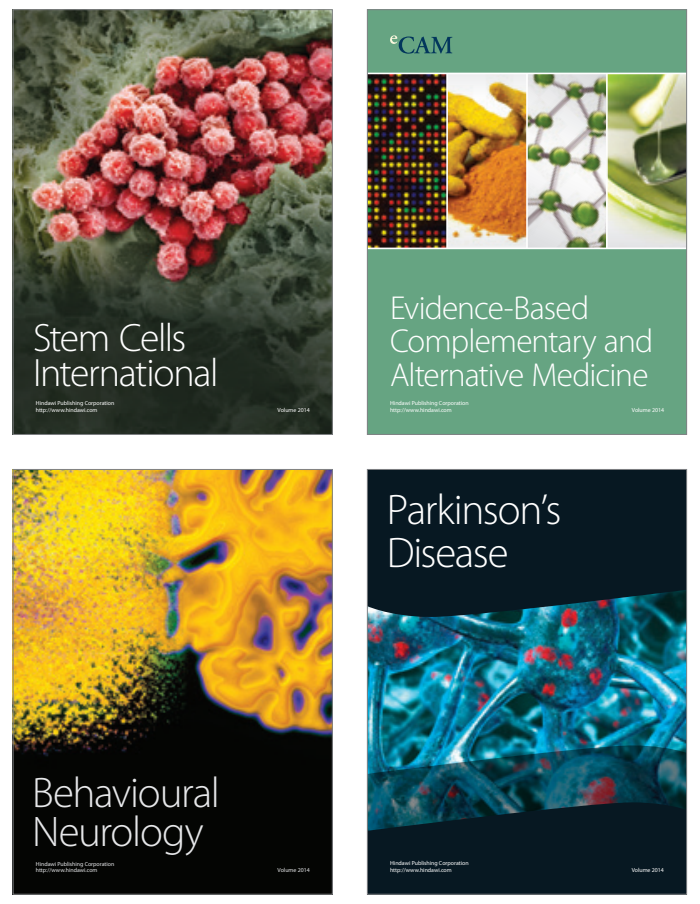
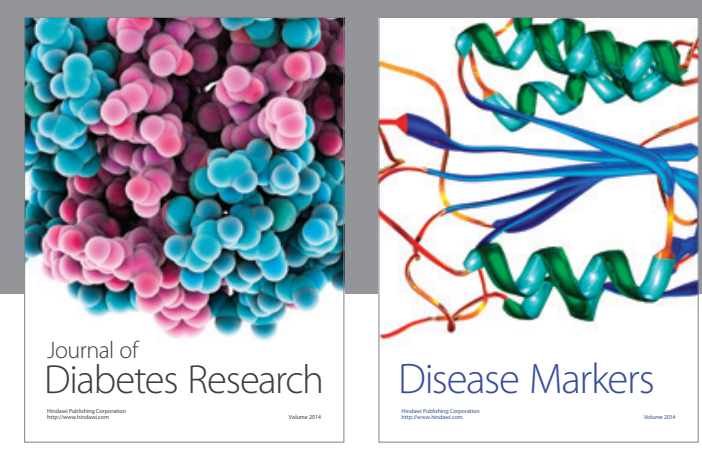

Disease Markers
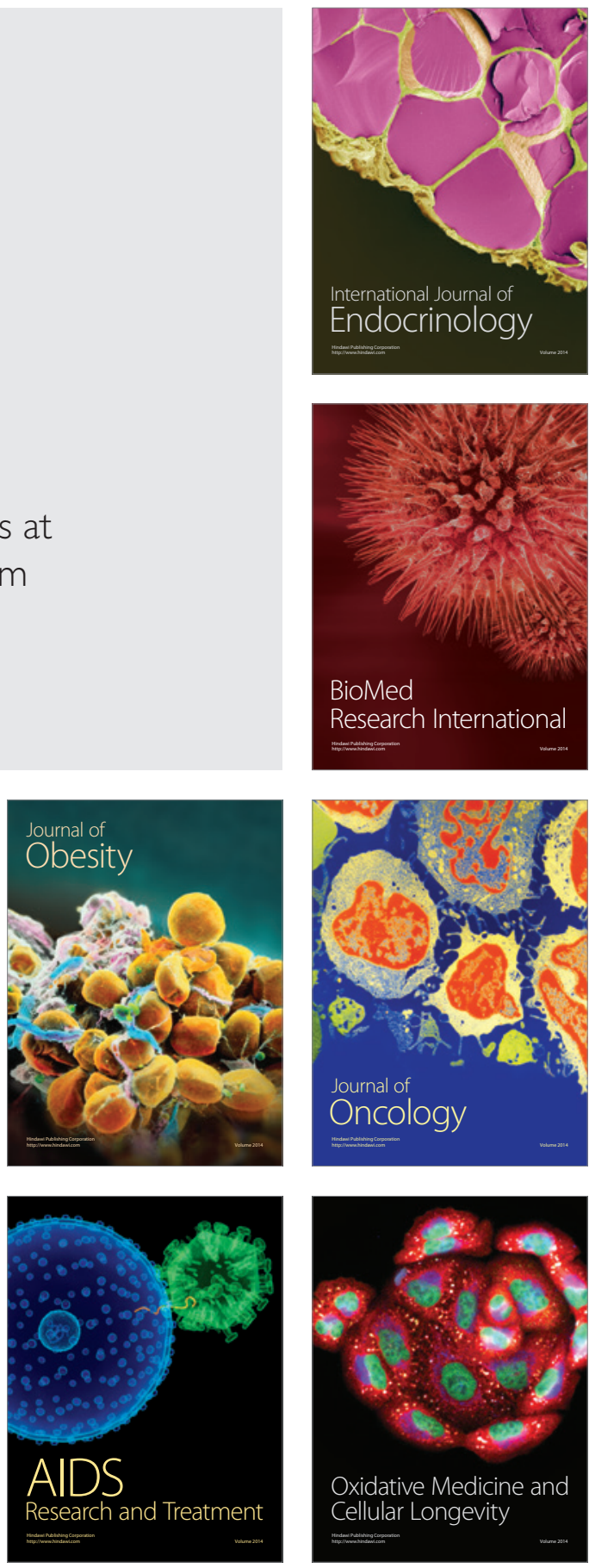